\title{
Single Electron Transistor: ApPlications \& PROBLEMS
}

\author{
Om Kumar ${ }^{1}$ and Manjit Kaur ${ }^{2}$ \\ ${ }^{1,2}$ VLSI-ES Department, Centre for Development of Advanced Computing, Mohali, India \\ om.prashar@gmail.com \\ mangur.k@gmail.com
}

\begin{abstract}
The goal of this paper is to review in brief the basic physics of nanoelectronic device single-electron transistor [SET] as well as prospective applications and problems in their applications. SET functioning based on the controllable transfer of single electrons between small conducting "islands". The device properties dominated by the quantum mechanical properties of matter and provide new characteristics coulomb oscillation, coulomb blockade that is helpful in a number of applications. SET is able to shear domain with silicon transistor in near future and enhance the device density. Recent research in SET gives new ideas which are going to revolutionize the random access memory and digital data storage technologies.
\end{abstract}

\section{Keywords}

Nanoelectronics; Single-electron transistor; Coulomb blockade, Coulomb oscillation, Quantum dot

\section{BASIC PHYSICS OF SET OpERATION}

Single Electron Transistor [SET] have been made with critical dimensions of just a few nanometer using metal, semiconductor, carbon nanotubes or individual molecules. A SET consist of a small conducting island [ Quantum Dot] coupled to source and drain leads bytunnel junctions and capactively coupled to one or more gate. Unlike Field Effect transistor, Single electron device based on an intrinsically quantum phenomenon, the tunnel effect. The electrical behaviour of the tunnel junction depends on how effectively barrier transmit the electron wave, which decrease exponentially with the thickness and on the number of electron waves modes that impinge on the barrier, which is given by the area of tunnel junction divided by the square of wave length.

Quantum dot [QD] is a mesoscopic system in which the addition or removal of a single electron can cause a change in the electrostatic energy or Coulomb energy that is greater than the thermal energy and can control the electron transport into and out of the QD. This sensitivity to individual electrons has led to electronics based on single electrons. For QD, the discrete energy level of the electrons in the QD becomes pronounced, like those in atoms and molecules, so one can talk about "artificial atoms and molecules". When the wave functions between two quantum dots overlap, the coupled quantum dots exhibit the properties of a molecule. To understand the electron transport properties in QD. Let us consider a metal nanoparticle sandwiched between two metal electrodes shown in figure 1 . The nanoparticle is separated from the electrodes by vacuum or insulation layer such as oxide or organic molecules so that only tunneling is allowed between them. So we can model each of the nanoparticles-electrode junctions with a resistor in parallel with a capacitor. The resistance is determined by the electron tunneling and the capacitance depends on the size of the particle. We denote the resistors and capacitors by $R_{1}, R_{2}$, $\mathrm{C}_{1}$ and $\mathrm{C}_{2}$, and the applied voltage between the electrodes by V. We will discuss how the current, I depends on V. When we start to increase V from zero, no current can flow between the electrodes because movement of an electron onto (charging) or off (discharging) from an initially neutral nanoparticle cost energy by an amount given by equation 1 . 


$$
E=e^{2} / 2 c
$$

This suppression of electron flow is called Coulomb blockade. Current start to flow through the nanoparticles only when the applied voltage V is large enough to establish a voltage $\varphi$ at the nanoparticles such that

$$
e \varphi \geq E=\frac{e^{2}}{2 C}
$$

This voltage is called threshold voltage and denoted by $\mathrm{V}_{\text {th }}$. So in the I-V curve, we expect a flat zero-current regime with a width of $2 \mathrm{~V}_{\text {th }}$. When the applied voltage reaches $\mathrm{V}_{\text {th }}$, an electron is added to (removed from) the nanoparticles. Further increasing the voltage, the current does not increase proportionally because it requires us to add (or remove) two electrons onto the nanoparticles, which cost a greater amount of energy. Once the applied voltage is large enough to overcome the Coulomb energy of two electrons, the current starts to increase again. This leads to a stepwise increase in I-V curve, called Coulomb staircase.

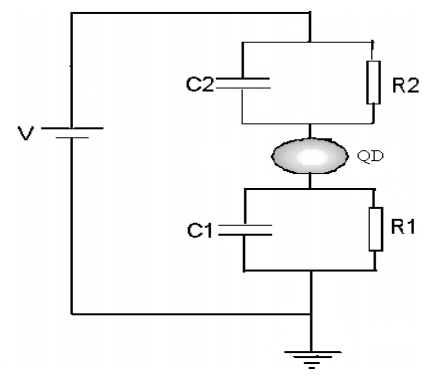

Figure 1. Quantum Dot Structure

\section{THEORETICAL BACKGROUND}

Throughout the history of single-electronics "orthodox" theory pioneered by Kulik and Shekhter play a very important role in understanding the behaviour of single electron devices. This is a very generalize theory based on the following major assumptions:

$\boldsymbol{A}$. The electron energy quantization inside the conductors is ignored, i.e. the electron energy spectrum is treated as continuous. This assumption is valid only if $\mathrm{E}_{\mathrm{k}}<<\mathrm{k}_{\mathrm{B}} \mathrm{T}$, but it frequently gives an adequate description of observations as soon as $\mathrm{E}_{\mathrm{k}}<<\mathrm{E}_{\mathrm{c}}$.

$\boldsymbol{B}$. The time $\tau_{\mathrm{t}}$ of electron tunneling through the barrier is assumed to be negligibly small in comparison with other time scales (including the interval between neighbouring tunneling events). This assumption is valid for tunnel barriers used in single-electron devices of practical interest, Where $\tau_{\mathrm{t}} \sim 10^{-15} \mathrm{~s}$.

C. Coherent quantum processes consisting of several simultaneous tunneling events ("cotunneling") are ignored. This assumption is valid if the resistance $\mathrm{R}$ of all the tunnel barriers of the system is much higher than the quantum unit of resistance $R_{Q}: R>R_{Q}$,

$$
\mathrm{R}_{\mathrm{Q}}=\mathrm{h} / 4 \mathrm{e}^{2} \approx 6.5 \mathrm{k} \Omega
$$

The orthodox theory is in quantitative agreement with virtually all the experimental data for systems with metallic conductors (with their small values of the electron wavelength on the Fermi surface, $\lambda_{F}$ and gives at least a qualitative description of most results for most 
semiconductor structures (where the quantization effects are more noticeable due to larger $\lambda_{\mathrm{F}}$ ). These assumptions are followed throughout the study of single electron systems.

\section{APPLICATIONS OF SET}

\subsection{Supersensitive Electrometer}

The high sensitivity of single-electron transistors have enabled them as electrometers in unique physical experiments. For example, they have made possible unambiguous observations of the parity effects in superconductors. Absolute measurements of extremely low dc currents $\left(\sim 10^{-20}\right.$ A) have been demonstrated. The transistors have also been used in the first measurements of single-electron effects in single-electron boxes and traps A modified version of the transistor has been used for the first proof of the existence of fractional-charge excitations in the fractional quantum hall effect.

\subsection{Single-Electron Spectroscopy}

One of the most important application of single-electron electrometry is the possibility of measuring the electron addition energies (and hence the energy level distribution) in quantum dots and other nanoscale objects.

\subsection{Current Standards}

One of the possible applications of single-electron tunneling is fundamental standards of dc current for such a standard a phase lock SET oscillations or Bloch oscillations in a simple oscillator with an external RF source of a well characterized frequency f. The phase locking would provide the transfer of a certain number $m$ of electrons per period of external RF signal and thus generate de current which is fundamentally related to frequency as $\mathrm{I}=m e f$. This arrangement have limitation of coherent oscillation that are Later overcome by the use of such a stable RF source to drive devices such as single-electron turnstiles and pumps, which do not exhibit coherent oscillations in the autonomous mode.

\subsection{Temperature Standards}

One new avenue toward a new standard of absolute temperature can be developed by the use of 1D single-electron arrays. At low temperatures, arrays with $\mathrm{N}>>1$ islands exhibit dc I-V curves generally similar to those of single-electron transistors with a clear Coulomb blockade of tunneling at low voltages $\left(|\mathrm{V}|<\mathrm{V}_{\mathrm{t}}\right)$ and approaching the linear asymptote $\mathrm{V}=\mathrm{NRI}+$ constant at $\left(|\mathrm{V}| \gg \mathrm{V}_{\mathrm{t}}\right)$. If the temperature is raised above $\mathrm{E}_{\mathrm{c}} / \mathrm{k}_{\mathrm{B}}$, thermal fluctuations smear out the Coulomb blockade, and the I-V curve is almost linear at all voltages: $G \equiv d V d V \approx G_{n} \equiv 1 / N R$ The only remaining artifact of the Coulomb blockade is a small dip in the differential conductance around $\mathrm{V}=0$.

\subsection{Detection of Infrared Radiation}

The calculations of the photo response of single-electron systems to electromagnetic radiation with frequency $\sim \mathrm{E}_{\mathrm{c}}$ h have shown that generally the response differs from that the well-known Tien-Gordon theory of photon-assisted tunneling. In fact, this is based on the assumption of independent (uncorrelated) tunneling events, while in single-electron systems the electron transfer is typically correlated. This fact implies that single-electron devices, especially 1D multi-junction array with their low co-tunneling rate, may be used for ultra-sensitive video- and heterodyne detection of high frequency electromagnetic radiation, similar to the superconductor-insulator-superconductor (SIS) junctions and arrays. The Single electron array have advantages over their SIS counterparts: Firstly lower shot noise and secondly convenient 
adjustment of the threshold voltage. This opportunity is especially promising for detection in the few-terahertz frequency region, where no background-radiation-limited detectors are yet available.

\subsection{Voltage State Logics}

The single-electron transistors can be used in the "voltage state" mode. In this mode, the input gate voltage $U$ controls the source-drain current of the transistor which is used in digital logic circuits, similarly to the usual field-effect transistors (FETs). This means that the single-electron charging effects are confined to the interior of the transistor, while externally it looks like the usual electronic device switching multi-electron currents, with binary unity/zero presented with high/low dc voltage levels (physically not quantized). This concept simplifies the circuit design which may ignore all the single-electron physics particulars. One substantial disadvantage of voltage state circuits is that neither of the transistors in each complementary pair is closed too well, so that the static leakage current in these circuits is fairly substantial, of the order of $10^{-4}$ e/RC. The corresponding static power consumption is negligible for relatively large devices operating at helium temperatures. However, at the prospective room-temperature operation this power becomes on the order of $10^{-7}$ Watt per transistor. Though apparently low, this number gives an unacceptable static power dissipation density $\left(>10 \mathrm{~kW} / \mathrm{cm}^{2}\right)$ for the hypothetical circuits which would be dense enough $\left(>10^{11}\right.$ transistors per $\left.\mathrm{cm}^{2}\right)$ to present a real challenge for the prospective CMOS technology.

\subsection{Charge State Logics}

The problem of leakage current is solved by the use of another logic device name charge state logic in which single bits of information are presented by the presence/absence of single electrons at certain conducting islands throughout the whole circuit. In these circuits the static currents and power vanish, since there is no dc current in any static state.

\subsection{Programmable Single Electron Transistor Logic}

An SET having non volatile memory function is a key for the programmable SET logic. The half period phase shift makes the function of SET complimentary to the conventional SETs. As a result SETs having non-volatile memory function have the functionality of both the conventional (n-MOS like) SETs and the complementary (p-MOS like) SETs. By utilising this fact the function of SET circuit can be programmed, on the basis of function stored by the memory function. The charged around the QD of the SET namely an SET island shift the phase of coulomb oscillation, the writing/erasing operation of memory function which inject/eject charge to/from the memory node near the SET island, makes it possible to tune the phase of coulomb oscillation. If the injected charge is adequate the phase shift is half period of the coulomb oscillation.

\section{Problems in SET ImPlementations}

\subsection{Lithography Techniques}

The first biggest problem with all single-electron logic devices is the requirement $E_{c} \sim 100 \mathrm{k}_{\mathrm{B}} \mathrm{T}$, which in practice means sub-nanometer island size for room temperature operation. In VLSI circuits, this fabrication technology level is very difficult. Moreover, even if these islands are fabricated by any sort of nanolithography, their shape will hardly be absolutely regular. Since in such small conductors the quantum kinetic energy gives a dominant contribution to the electron addition energy $\left(E_{k}>E_{c}\right.$, ), even small variations in island shape will lead to unpredictable and rather substantial variations in the spectrum of energy levels and hence in the device switching thresholds. 


\subsection{Background Charge}

The second major problem with single-electron logic circuits is the infamous randomness of the background charge. A single charged impurity trapped in the insulating environment polarizes the island, creating on its surface an image charge $\mathrm{Q}_{0}$ of the order of e. This charge is effectively subtracted from the external charge $\mathrm{Q}_{\mathrm{e}}$

\subsection{Cotunneling}

The essence of the effect is that the tunneling of several $(\mathrm{N}>1)$ electrons through different barriers at the same time is possible as a single coherent quantum-mechanical process. The rate of this process is crudely $(\mathrm{RQ} / \mathrm{R}) \mathrm{N}-1$ times less than that for the single-electron tunneling described by Equation of the orthodox theory

$$
\Gamma(\Delta W)=(1 / e) I(\Delta W / e)[1-\exp \{-\Delta W / k B T\}]-1
$$

If the condition expressed by equation (3) is satisfied this ratio is rather small; cotunneling can nevertheless be clearly observed within the Coulomb blockade range where orthodox tunneling is suppressed.

\subsection{Room Temperature Operation}

The first big problem with all the known types of single-electron logic devices is the requirement $E_{\mathrm{c}} \sim 100 k_{B} T$, which in practice means sub-nanometer island size for room temperature operation. in such small conductors the quantum kinetic energy gives a dominant contribution to the electron addition energy even small variations in island shape will lead to unpredictable and rather substantial variations in the spectrum of energy levels and hence in the device switching thresholds.

\subsection{Linking SETs with the Outside Environment}

The individual structures patterns which function as logic circuits must be arranged into larger 2D patterns. There are two ideas. The first is to integrating SET as well as related equipments with the existed MOSFET, this is attractive because it can increase the integrating density. The second option is to give up linking by wire, instead utilizing the static electronic force between the basic clusters to form a circuit linked by clusters, which is called quantum cellular automata (QCA). The advantage of QCA is its fast information transfer velocity between cells (almost near optic velocity) via electrostatic interactions only, no wire is needed between arrays and the size of each cell can be as small as $2.5 \mathrm{~nm}$, this made them very suitable for high density memory and the next generation quantum computer.

\section{Conclusions}

Single Electronic Transistor (SET) has proved their value as tool in scientific research. Resistance of SET is determined by the electron tunneling and the capacitance depends on the size of the nanoparticle. The current starts to flow through the junction when applied voltage is just sufficient to raise the energy of electron above the coulomb blocked, this is called threshold voltage $V_{\text {th }}$ and the flat zero current persist for $2 V_{\text {th }}$. Several applications of nanoscale devices in metrology, including the fundamental standards of current, resistance and temperature also seem quite promising. Another potential application is terahertz radiation detection. The situation is much more complex with digital single electronics. The concept of single electron logic suggested so far face sturdy challenges: either removing background charge or providing continuous charge transfer in nanoscale. The main problem in nanometer era is the fabrication 
of nanoscale devices. SET provide the potential for low-power, intelligent LSI chips, appropriate for ubiquitous application.

\section{REFERENCES}

[1] M. A. Kastner, "The single electron transistor and artificial atoms”, Ann. Phy. (Leipzig), vol. 9, pp. 885-895, 2000.

[2] S. Bednarek, B. Szafran, and J. Adamowski, "Solution of the Poisson Schrodinger problem for a single-electron transistor”, Phys. Rev. B, Vol. 61, pp. 4461-4464, 2000.

[3] Songphol Kanjanachuchai and Somsak Panyakeow, "Beyond CMOS: Single-Electron Transistors", IEEE International Conference on Industrial Technology, Bangkok, Thailand, 2002.

[4] Masumi Saitoh, Hidehiro Haratalion and Toshiro Hiramoto, "Room-Temperature Demonstration of Integrated Silicon Single-Electron Transistor Circuits for Current Switching and Analog Pattern Matching", IEEE International Electron Device Meeting, San Francisco, USA, 2004.

[5] K. Matsumoto, M. Ishii, K. Segawa, Y. Oka B. J. Vartanian and J. S. Harris, "Room temperature operation of a single electron transistor made by the scanning tunneling microscope nano oxidation process for the TiOx/Ti system”, Appl. Phys. Lett. 68 (1), pp. 34-36, 1996.

[6] Ken Uchida, Jugli Kaga, Ryuji Ohba and Akira Toriumi, "Programmable Single-Electron Transistor Logic for Future Low-Power Intelligent LSI: Proposal and Room-Temperature Operation”, IEEE Transactions on Electron Devices, Vol. 50, No. 7, July 2003.

[7] T.A. Fulton and G.D. Dolan, "Observation of single electron charging effect in small tunnelling junction”, Phys. Rev. Lett., Vol. 59, pp. 109-112, July 1987.

[8] Lingjie Guo, Effendi Leobandung and Stephen Y. Chou, “A silicon Single-Electron transistor Memory operating at room temperature”, Science Vol. 275, pp. 649-651, 1997.

[19] A.N. Cleand, D. Estene, C. Urbina and M.H. Devoret, "An extremely Low noise Photodetector based on the single electron Transistor" , Journal of Low Temperature Physics, Vol. 93, Nos. 3/4, pp.767-772, 1993.

[10] R. Knobel, C.s. Yung and A.N. clelanda, "Single -electron transistor as a radio frequency mixer" , Applied Physics Letters, Vol. 81, No. 3, pp. 532-534, July 2002.

\section{Authors}

Om Kumar ${ }^{1}$ I am a Student of Masters in Technology in VLSI Design, Centre for Development of Advanced Computing(C-DAC), Mohali, India. My research interests include Semiconductor Device Physics, Nano electronics.

Manjit Kaur ${ }^{2}$ I am Bachelor in Electronics and communication Engineering, BECT Gurdaspur,Punjab, India and Masters in Technology in Microelectronics from Punjab University, Chandigarh, India. Presently working as Design Engineer at Centre for Development of Advanced Computing(C-DAC), Mohali, India. This institute is a scientific society of the Ministry of Communications \& Information Technology, Govt. of India, involved in Research and Development with an objective to create focus on Advanced Information Technologies, High-end Academics \& Training

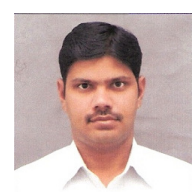
relevant to $\mathrm{R} \& \mathrm{D}$ societies. I am associated with Masters in Technology in VLSI Design. My research interests include VLSI design, Low power VLSI Design techniques, Semiconductor Device Physics, Nano electronics. 